\title{
An automatic holographic adaptive phoropter
}

Babak Amirsolaimani, N. Peyghambarian, Jim Schwiegerling, Arkady Bablumyan, Nickolaos Savidis, et al.

Babak Amirsolaimani, N. Peyghambarian, Jim Schwiegerling, Arkady Bablumyan, Nickolaos Savidis, Gholam Peyman, "An automatic holographic adaptive phoropter," Proc. SPIE 10352, Biosensing and Nanomedicine X, 1035208 (29 August 2017); doi: 10.1117/12.2276807

SPIE Event: SPIE Nanoscience + Engineering, 2017, San Diego, California, United States 


\title{
An Automatic Holographic Adaptive Phoropter
}

\author{
Babak Amirsolaimani, N. Peyghambarian, Jim Schwiegerling, Arkady Bablumyan, Nickolaos \\ Savidis, Gholam Peyman* \\ College of Optical Sciences, University of Arizona, Tucson, AZ 85721, USA
}

\begin{abstract}
Phoropters are the most common instrument used to detect refractive errors. During a refractive exam, lenses are flipped in front of the patient who looks at the eye chart and tries to read the symbols. The procedure is fully dependent on the cooperation of the patient to read the eye chart, provides only a subjective measurement of visual acuity, and can at best provide a rough estimate of the patient's vision. Phoropters are difficult to use for mass screenings requiring a skilled examiner, and it is hard to screen young children and the elderly etc. We have developed a simplified, lightweight automatic phoropter that can measure the optical error of the eye objectively without requiring the patient's input. The automatic holographic adaptive phoropter is based on a Shack-Hartmann wave front sensor and three computercontrolled fluidic lenses. The fluidic lens system is designed to be able to provide power and astigmatic corrections over a large range of corrections without the need for verbal feedback from the patient in less than 20 seconds.
\end{abstract}

Keywords: Phoropter, Fluidic lens, Holographic Optical Element

\section{INTRODUCTION}

A phoropter is one of the most commonly used instruments by ophthalmologists and optometrists. The refractive error of the eye is measured using a set of lenses with power increments of 0.25 diopter. These lenses are placed one at a time in front of the patient's eye, while looking at an eye chart at some distant away. A large number of lenses is required to cover the most common vision errors of myopia (nearsightedness), hyperopia (farsightedness), and astigmatism within the required range. This will result in large physical footprint of the phoropter and adds to its complexity. Moreover, this process can take a long time and requires constant and reliable feedback from the patient, which can cause some errors in the actual prescribed data, especially for young children and infants who cannot have verbal communication.

Fluidic lenses are a good alternative to reduce the number of lenses required in a phoropter system. Continuous power change of these lenses is also another potential advantage that can lower the 0.25 diopter increments. Tunable lenses have been studied in different applications such as adaptive eyeglasses [1], confocal microscopy [2], microscope objectives [3, 4], and camera lenses $[5,6]$. Marks et al $[7,8]$ investigated application of fluidic lenses for ophthalmic correction, using manually pumped fluidic lenses to correct defocus and astigmatism errors. While these hydraulically pumped lenses have showed to support large correction range of -20 to 20 diopters, they suffer from lack of feedback system for automatic correction and large system footprint.

Various methods to fabricate and control liquid lenses have been investigated [9]. Liquid crystals [10], Electrowetting (EW) $[11,12]$, thermal stimulation [13], and external pressure actuated pumps [14-18] have all shown promising applications for tunable lenses. Although most these methods are limited to spherical powers, recent studies by Kope and Zappe [19], and Liebetraut et al [20] showed tunable astigmatism in liquid lenses, as well. The main disadvantage of using the EW lens designs is

*gpeyman1@yahoo.com

Biosensing and Nanomedicine $\mathrm{X}$, edited by Hooman Mohseni, Massoud H. Agahi, Manijeh Razeghi, Proc. of SPIE Vol. 10352, 1035208 - @ 2017 SPIE · CCC code: 0277-786X/17/\$18 - doi: 10.1117/12.2276807 
the limited movement range (limited change in focal length) which make them unsuitable for eye refractive correction.

In order to overcome the abovementioned problems, in this paper we demonstrate a new compact fluidic lens based auto-phoropter. The fluidic lens membranes are made of Polydimethylsiloxane (PDMS) and the correction power is controlled using hydraulic pump. By choosing the appropriate mechanical aperture, these lenses can be utilized for both defocus and astigmatism correction. Moreover, holographic optical elements (HOEs) [21] are deployed to provide an unobstructed view for the patient during the measurement in the visible wavelengths while redirecting the IR light into the wavefront sensor. The system design and simulations are presented in next part following by the fabricated setup test results.

\section{PHOROPTER DESIGN}

The auto-phoropter design is depicted in Figure 1A. Green color shows the visible light path in the system while orange illustrated the infrared light traveling form the source to the detector. The collimated 780nm laser steers towards the eye using 50:50 beam splitter. The light passes through the fluidic lens setup before reaching the eye. The first two fluidic lenses have cylindrical shape (place at 45 degrees with respect to each other) to correct astigmatism at different angles while the third one has spherical shape to correct the defocus. After passing through the three fluidic lenses, the reflect light from the retina goes through the HOE telescope system. The HOE telescope system is designed to image the eye pupil on to the wavefront detector and changing the beam size to fit the detector's aperture. Moreover, the HOEs are designed specifically for $780 \mathrm{~nm}$ wavelength and they don't affect the visible wavelength range. Hence, patient's view will not be blocked while looking at a distant eye chart and the eye is not blinded by the visible laser light. A Shack-Hartmann detector is used to measure the Zernike values and optometric constants of the IR wavefront. The fluidic lenses are controlled using hydraulic pumps based on the feedback data from the Shack-Hartmann detector. Figure 1B and $1 \mathrm{C}$ show the fabricated compact phoropter system and the fluidic lenses respectively.

To fabricate the lens membranes, Sylgard-184 PDMS form Dow Corning was spin coated at on a two-inch flat glass substrate and baked in the oven at $100^{\circ} \mathrm{C}$ for 30 minutes. The thickness of the PDMS membranes are measured using a DEKTAK profilometer. Using 500 RPM spin coating for 15 seconds and degassing the PDMS before bake, creates a $250 \mu \mathrm{m}$ thick uniform membrane. Laser liquid with refractive index of 1.55 is used as the fluid inside the lenses to minimize the index mismatch between the rigid glass side, the fluid, and the deformable PDMS membrane of each lens. By choosing appropriate dimensions for the circular (radius $=10 \mathrm{~mm}$ ) and rectangular (width $=$ $13 \mathrm{~mm}$, height $=28 \mathrm{~mm}$ ) clamps for the membranes, the lenses would act as spherical and cylindrical within the optical aperture of the setup (radius $=5 \mathrm{~mm}$ ). Moreover, refractive correction can be achieved within the range of 20 diopters at different angles.

The clear aperture of the lenses are $10 \mathrm{~mm}$ in diameter. The mechanical clamps are chosen such that the lenses behave as spherical or cylindrical within the optical aperture. The deformation of the PDMS membranes can be modeled as a quadratic polynomial [22]. Membranes were modeled as even aspherical and toroidal surfaces in Zemax for spherical and cylindrical lenses respectively. By changing the curvature form $-0.024 / \mathrm{mm}$ to $0.024 / \mathrm{mm}$, the focal length can be changed from -100 to $100 \mathrm{~mm}$ (-10 to 10 diopters). Simulated Zernike value of defocus (Z4), 45 ${ }^{\circ}$ astigmatism (Z5), and $90^{\circ}$ astigmatism (Z6) at two different lens powers $(-5$ and +5 diopters $)$ in the spherical and 
cylindrical lenses is represented in Figure2A. The volume change measurement is based on the surface SAG variations of the spherical cap and cylindrical segments. The spherical and cylindrical optometric constants (in diopters) and the axis angle of the cylinder in degrees can be calculated based on these Zernike values [23].
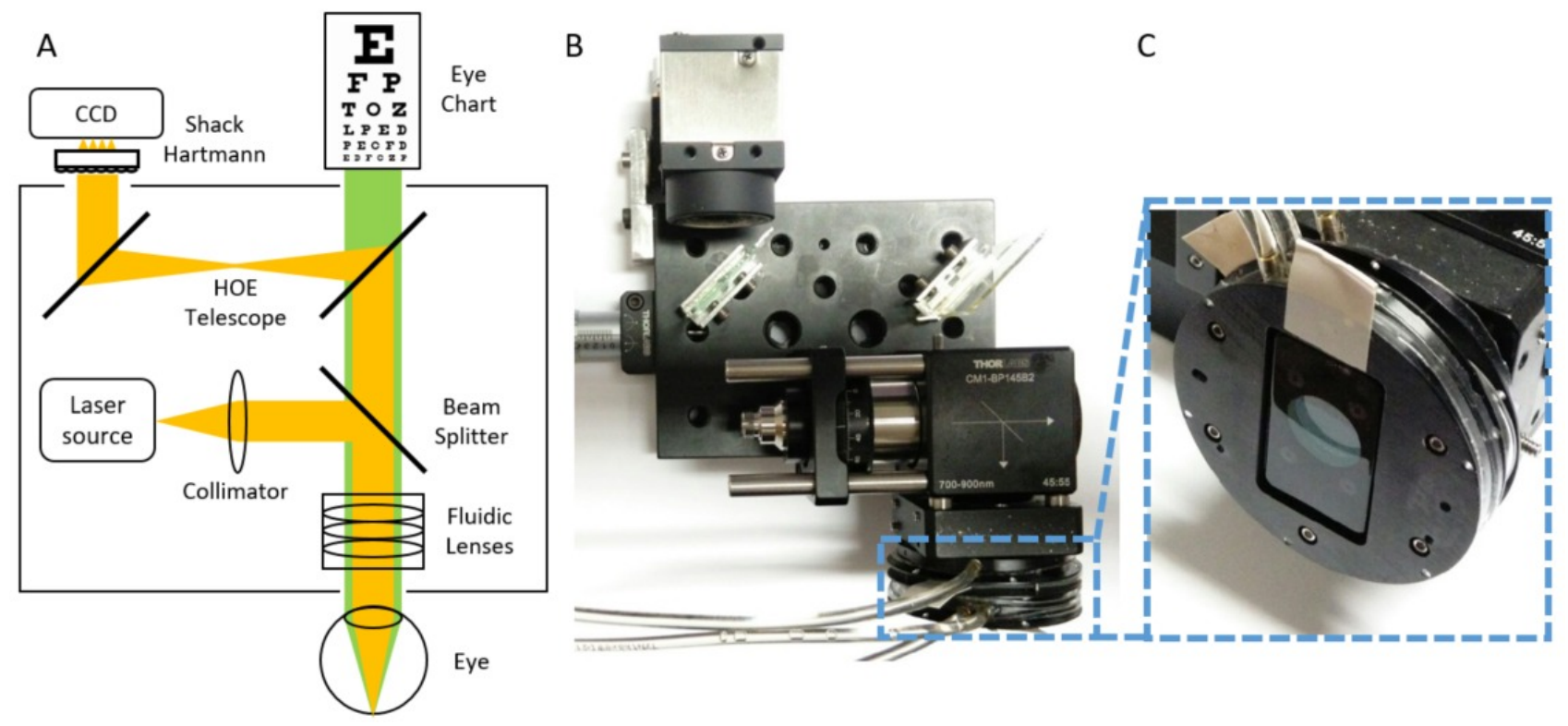

Figure 1: A) The schematic diagram of the auto-phoropter system. Green Color represents the visible light path from the eye chart to the patient's eye. Orange color illustrates the 780nm laser light traveling from the source to the eye and finally to the detector. B) The image of the holographic optical elements, fluidic lenses, and the fabricated compact phoropter setup. C) The zoomed in image of the fluidic lenses. Three separate fluidic lenses are used in the setup to control the defocus and astigmatism at different angles. The magnification power of each lens is controlled using a high-resolution hydraulic pump.

\section{RESULTS}

The fluidic lenses are characterized using their Zernike values at different magnifications. Shack-Hartmann sensor was used to measure the Zernike values for defocus (Z4), oblique astigmatism (Z5), and vertical astigmatism (Z6) at different dioptric lens powers. Figure $2 \mathrm{~B}$ shows the normalized measured result for each lens at -5 and +5 diopters. As can be seen the measured results match the Zemax model quite well, which shows the fluidic lenses can be replaced the traditional spherical and cylindrical lenses of a the phoropter. The power of each lens adjusts using the feedback data form the in-house developed. Net software that calculates the required volume change based on the optometric constants of the infrared wavefront reaching the Shack-Hartmann detector.

Figure 3 illustrates the imaging results through the defocus lens at three different focal lengths $(10 \mathrm{~cm}, 40 \mathrm{~cm}$, and $100 \mathrm{~cm})$. By tuning this focal distance using a hydraulic pump, magnification power variation of -10 to 10 diopters with 0.1 diopter increments is achieved. The same magnification control is also applied for the cylindrical lenses to control the astigmatism at different angles. 


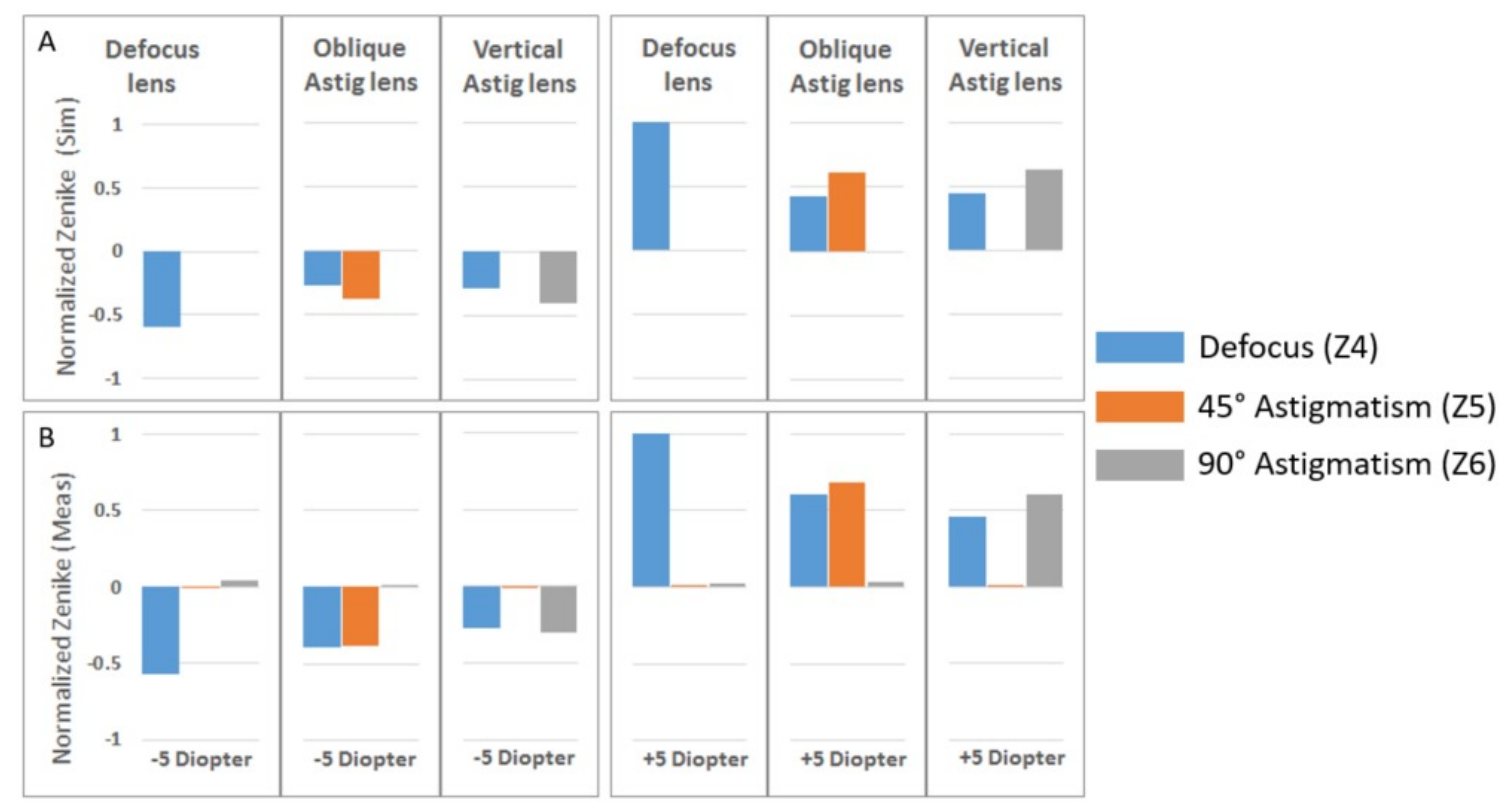

Figure 2: A) Zemax simulation results of normalized Zernike values at -5 and +5 diopter for defocus, oblique astigmatism and vertical astigmatism lenses. B) The measured results of the corresponding Zernike values for each lens. The measured results matches quite well with simulations, which shows that the defocus lens can be modeled as a spherical surface and cylindrical surface modeling can be used for astigmatic lenses.
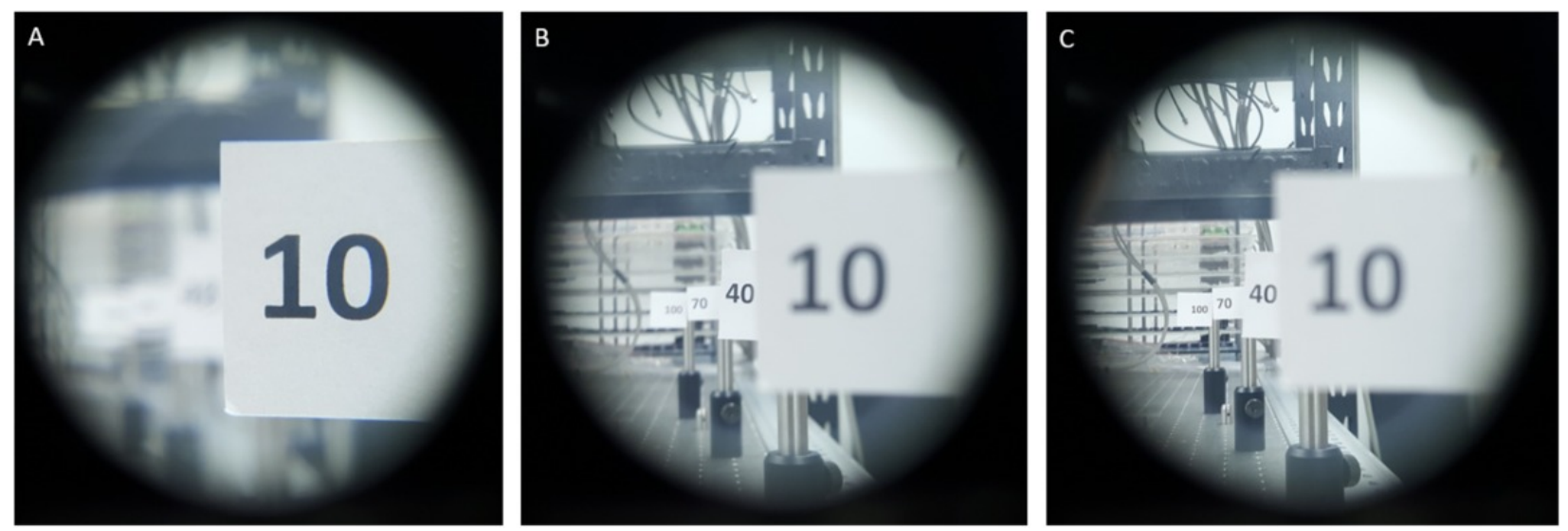

Figure 3: Magnification changes of the defocus fluidic lens depicted at A) $10 \mathrm{~cm}, B) 40 \mathrm{~cm}$, and C) $100 \mathrm{~cm}$ focal length. By changing the volume of the fluid, the lens power can be tuned to the desired dioptric value. The magnification change of -10 to +10 diopter with 0.1 diopter increments can be achieved using the hydraulically pumped fluidic lens.

\section{CONCLUSION}

In this paper, we introduced a compact auto-phoropter system. The refractive correction using PDMS based tunable fluidic lenses showed high accuracy for spherical aberration and astigmatism. Power range of -10 to +10 diopters was achieved using hydraulically actuated pumps with high resolution. 3D printing of the pumps and lenses on the same structure can further reduce the system footprint while increasing the accuracy and response time of the system. Moreover, employing holographic optical elements made use of their unique property in steering the IR light towards the detector without affecting the patient's line of sight in visible range. Finally, automatic refractive power correction using the data form Shack-Hartmann detector eliminates the need for 
verbal feedback from the patient and increases the prescription accuracy while decreasing the examination time.

\section{REFERENCES}

[1] Nazmul Hasan, Aishwaryadev Banerjee, Hanseup Kim, and Carlos H. Mastrangelo, "Tunable-focus lens for adaptive eyeglasses," Opt. Express 25, 1221-1233 (2017)

[2] Minkyu Kim, DongKyun Kang, Tao Wu, Nima Tabatabaei, Robert W. Carruth, Ramses V Martinez, George M. Whitesides, Yoshikazu Nakajima, and Guillermo J. Tearney, "Miniature objective lens with variable focus for confocal endomicroscopy," Biomed. Opt. Express 5, 4350-4361 (2014)

[3] Gongpu Lan, Thomas F. Mauger, and Guoqiang Li, "Design of high-performance adaptive objective lens with large optical depth scanning range for ultrabroad near infrared microscopic imaging," Biomed. Opt. Express 6, 3362-3377 (2015)

[4] Lei Li, Di Wang, Chao Liu, and Qiong-Hua Wang, "Zoom microscope objective using electrowetting lenses," Opt. Express 24, 2931-2940 (2016)

[5] S. Kuiper and B. H. W. Hendriks, "Variable-focus liquid lens for miniature cameras," Appl. Phys. Lett. 85, 1128 (2004)

[6] Frank S. Tsai, Sung Hwan Cho, Yu-Hwa Lo, Bob Vasko, and Jeff Vasko, "Miniaturized universal imaging device using fluidic lens," Opt. Lett. 33, 291-293 (2008)

[7] Randall Marks, David L. Mathine, Gholam Peyman, Jim Schwiegerling, and Nasser Peyghambarian, "Adjustable fluidic lenses for ophthalmic corrections," Opt. Lett. 34, 515-517 (2009)

[8] Randall Marks, David L. Mathine, Gholam Peyman, Jim Schwiegerling, and N. Peyghambarian, "Adjustable adaptive compact fluidic phoropter with no mechanical translation of lenses," Opt. Lett. 35, 739-741 (2010)

[9] Kartikeya Mishra, Dirk van den Ende, and Frieder Mugele, "Recent Developments in Optofluidic Lens Technology," Micromachines 7, 102 (2016)

[10] Pouria Valley, Nickolaos Savidis, Jim Schwiegerling, Mohammad Reza Dodge, Gholam Peyman, and Nasser Peyghambarian, "Adjustable hybrid diffractive/refractive achromatic lens," Opt. Express 19, 7468-7479 (2011)

[11]B. Berge, J. Peseux, "Variable focal lens controlled by an external voltage: An application of electrowetting," Eur. Phys. J. E, 3 (2000)

[12] S. Kuiper, B.H.W. Hendriks, "Variable-focus liquid lens for miniature cameras,” Appl. Phys. Lett. 85 (2004)

[13] Wei Zhang, Hans Zappe, Andreas Seifert, "Wafer-scale fabricated thermo-pneumatically tunable microlenses," Light: Science \& Applications, 3 (2014)

[14] Campbell Kyle, Yeshaiahu Fainman, Alex Groisman, "Pneumatically actuated adaptive lenses with millisecond response time." Appl. Phys. Lett. 91 (2007)

[15] M. Blum, M. Büeler, C. Grätzel, J. Giger, M. Aschwanden, "Optotune focus tunable lenses and laser speckle reduction based on electroactive polymers", Proc. SPIE 8252, MOEMS and Miniaturized Systems XI, (2012)

[16] Hongwen Ren, David Fox, P. Andrew Anderson, Benjamin Wu, and Shin-Tson Wu, "Tunable-focus liquid lens controlled using a servo motor," Opt. Express 14, 8031-8036 (2006)

[17] Nickolaos Savidis, Gholam Peyman, Nasser Peyghambarian, and Jim Schwiegerling, "Nonmechanical zoom system through pressure-controlled tunable fluidic lenses," Appl. Opt. 52, 2858-2865 (2013)

[18] De-Ying Zhang, Victor Lien, Yevgeny Berdichevsky, Jaehyuck Choi, and Yu-Hwa Lo, "Fluidic adaptive lens with high focal length tunability," Appl. Phys. Lett. 82, 3171 (2003)

[19] Daniel Kopp and Hans Zappe, "Tubular astigmatism-tunable fluidic lens," Opt. Lett. 41, 2735-2738 (2016)

[20] Peter Liebetraut, Sebastian Petsch, Jens Liebeskind and Hans Zappe, "Elastomeric lenses with tunable astigmatism," Light: Science \& Applications 2 (2013)

[21] Arkady Bablumyan, Pierre-Alexandre Jean Blanche, Nasser N. Peyghambarian, "System for holography," US Patent US8634119 B2 (2014)

[22] Xiang Qian, Wenhui Zhang, Cheng Peng, Xingyang Liu, Quan Yu, Kai Ni, and Xiaohao Wang, "Characterizing the Deformation of the Polydimethylsiloxane (PDMS) Membrane for Microfluidic System through Image Processing," Micromachines 7, 92 (2016)

[23] James C. Wyant, and Katherine Creath, "Basic wavefront aberration theory for optical metrology," Applied optics and optical engineering 11.s 29 (1992) 